\title{
Correction to: A year in type 2 diabetes mellitus: 2018 review based on the Endorama lecture
}

\section{Stavroula A. Paschou ${ }^{1}$. Theodoros Alexandrides ${ }^{2}$}

Published online: 9 January 2020

(C) Hellenic Endocrine Society 2019

\section{Correction to: Hormones}

https://doi.org/10.1007/s42000-019-00139-z

The original version of this article, published on 26 November 2019, contained a mistake. An author's name was misspelled. The correct author name reads as follows:

Stavroula A. Paschou

The original article has been corrected.

The online version of the original article can be found at https://doi.org/ $10.1007 / \mathrm{s} 42000-019-00139-\mathrm{z}$

Stavroula A. Paschou s.a.paschou@gmail.com

1 Division of Endocrinology and Diabetes, "Aghia Sophia" Hospital, Medical School, National and Kapodistrian University of Athens, Athens, Greece

2 Department of Endocrinology, University Hospital, Medical School, University of Patras, Rio, Patras, Greece 